\title{
Maternal creatine supplementation during pregnancy prevents acute and long-term deficits in skeletal muscle after birth asphyxia: a study of structure and function of hind limb muscle in the spiny mouse
}

\author{
Domenic A. LaRosa', Stacey J. Ellery' ${ }^{1}$ Rod J. Snow², David W. Walker' and Hayley Dickinson'
}

BACKGROUND: Maternal antenatal creatine supplementation protects the brain, kidney, and diaphragm against the effects of birth asphyxia in the spiny mouse. In this study, we examined creatine's potential to prevent damage to axial skeletal muscles.

METHODS: Pregnant spiny mice were fed a control or creatine-supplemented diet from mid-pregnancy, and $1 \mathrm{~d}$ before term (39 d), fetuses were delivered by c-section with or without $7.5 \mathrm{~min}$ of birth asphyxia. At $24 \mathrm{~h}$ or $33 \pm 2 \mathrm{~d}$ after birth, gastrocnemius muscles were obtained for ex-vivo study of twitch-tension, muscle fatigue, and structural and histochemical analysis. RESULTS: Birth asphyxia significantly reduced cross-sectional area of all muscle fiber types $(P<0.05)$, and increased fatigue caused by repeated tetanic contractions at $24 \mathrm{~h}$ of age $(P<$ $0.05)$. There were fewer $(P<0.05)$ Type I and lla fibers and more $(P<0.05)$ Type $\mathrm{llb}$ fibers in male gastrocnemius at $33 \mathrm{~d}$ of age. Muscle oxidative capacity was reduced $(P<0.05)$ in males at $24 \mathrm{~h}$ and $33 \mathrm{~d}$ and in females at $24 \mathrm{~h}$ only. Maternal creatine treatment prevented all asphyxia-induced changes in the gastrocnemius, improved motor performance.

CONCLUSION: This study demonstrates that creatine loading before birth protects the muscle from asphyxia-induced damage at birth.

$\mathbf{T}$ he third leading cause of neonatal death and morbidity worldwide is birth asphyxia (1), and it can be caused by a number of events including transient umbilical cord compression, placental abruption, or protracted labor (2). Underperfusion and reduced gas exchange in the placenta renders fetal tissues hypoxic, with metabolism often progressing to acidemia that compromises mitochondrial function, leading to cellular energy failure and accumulation of reactive oxygen and nitrogen species (ROS/RNS) (3).

A major physiological response of the fetus to hypoxia is the redistribution of blood flow to maintain adequate perfusion, and thus oxygenation, of the brain, heart, and adrenal glands (4). This reduces blood flow to the peripheral organs, such as the muscles of the trunk and legs, which, if severe or prolonged, may be sufficient to damage the respiratory apparatus (i.e., mitochondria) in such tissues. In adult muscle, hypoxia reduces the capacity for ATP production (5), with the muscle fibers becoming increasingly dependent on anaerobic energy production. This metabolic disturbance is associated with an accumulation of metabolic by-products such as ROS/RNS, thus contributing further to a deterioration of muscle fiber function, including increased fatigue (6). Excessive production of ROS and RNS can also promote the degradation of the myofibrillar proteins by increasing protease activity and upregulation of muscle-specific E3-ligases such as atrogin-1 and MuRF1 (7-9), resulting in reduced skeletal muscle function (10), and muscle fiber atrophy (11).

In addition to the primary global hypoxia-ischemia, skeletal muscle can endure a "second hit" when blood flow is reestablished, as reperfusion is associated with large increases in ROS and proinflammatory cytokine production (12), resulting in a second round of energy failure and further compromise of muscle fiber function (13).

Few studies have investigated the effect of a hypoxic episode at birth on skeletal muscle structure and function. As mentioned above, fetal cardiovascular responses to hypoxia are usually transient, but it is not certain that this is so when severe hypoxia occurs at birth. Previous work in our laboratory using the precocial spiny mouse found that the neonatal diaphragm showed significant changes to structure and functional deficits after birth asphyxia (14), and we have recently reported that this damage persists for at least $33 \mathrm{~d}$ after birth, an age equivalent to adolescence in this species (15).

If similar effects occurred in the axial and trunk muscles, this might contribute to the hypotonia present in some neonates, and the limited physical activity that is often displayed by infants who recover from asphyxia at birth. Cerebral palsy

\footnotetext{
The last two authors are the joint senior authors

${ }^{1}$ Ritchie Centre, Hudson Institute of Medical Research and Department of Obstetrics and Gynaecology, Monash University, Melbourne, Australia; ${ }^{2}$ Centre for Physical Activity and Nutrition, Deakin University, Burwood, Australia. Correspondence: Domenic A. LaRosa (domenic.larosa@hudson.org.au)

Received 9 March 2016; accepted 10 June 2016; advance online publication 24 August 2016. doi:10.1038/pr.2016.153
} 
(CP), an outcome often attributed to antenatal/intrapartum hypoxia and birth asphyxia (16), is characterized by hypotonia in the immediate neonatal period, and ultimately leads to deficits in motor co-ordination, alterations in gait $(17,18)$, and muscle spasticity in severe cases (19). Further outcomes may include significant reductions in mean and peak muscle power, decreased limb muscle mass $(2,20)$, reductions in muscle fiber size, and alterations in fiber type proportions $(3,18,20)$. The question of whether these muscular deficiencies arise due to neuronal damage and changes in innervation, and therefore as a "disuse phenotype" due to inactivity $(4,21)$, or are a consequence of primary injury to the muscle itself, remains unanswered. Furthermore, treatments currently available are limited to rehabilitation (e.g., physiotherapy) and Botulinum toxin $\mathrm{A}(5,22)$, rather than prevention.

Supplementation of the maternal diet with creatine $(\mathrm{Cr}$; $5 \% \mathrm{w} / \mathrm{w})$ in the latter half of pregnancy is protective for the brain $(6,23)$, kidney $(7-9,24)$, and diaphragm $(10,14)$ against the damaging effects of birth asphyxia in the spiny mouse. These organs have a high requirement for oxygen at birth and can be regarded as relatively intolerant to hypoxia. The Cr-phosphocreatine system endogenously maintains cellular energy turnover by acting as a spatial and temporal ATP shuttle, as well as providing an additional anaerobic source of ATP when oxidative phosphorylation fails. This led us to examine if the level of birth asphyxia known to compromise brain, renal and diaphragm structure and function also affected a large axial muscle in this way, and if so, whether this compromised muscle structure and function persisted into early adult life. Therefore, we assessed the structural and functional effects of birth asphyxia in a representative, mixed fiber type axial skeletal muscle (hindlimb gastrocnemius muscle) in both the immediate neonatal period and in early adult life. Further, we examined if maternal creatine supplementation during the latter half of pregnancy could prevent such damage from occurring, using the spiny mouse as a small animal model of birth asphyxia.

We hypothesized that birth asphyxia would produce structural and functional deficits in the gastrocnemius muscle in the immediate neonatal period that would persist until adulthood. Furthermore, we postulated that these changes would be prevented by maternal dietary creatine supplementation.

\section{RESULTS}

\section{Total Creatine Content}

At $24 \mathrm{~h}$ after birth by c-section, the total creatine content in gastrocnemius muscle of offspring from creatine-fed dams was $35 \%$ higher than those from control-fed mothers $(111.9 \pm 4.4$ vs. $83.1 \pm 4.2 \mathrm{mmol} / \mathrm{g} /$ dry weight; $P<0.05$ ).

\section{Fiber Type Proportions in the Gastrocnemius Muscle}

Representative images of myofibrillar ATPase staining for each group at $24 \mathrm{~h}$ and $33 \mathrm{~d}$ are shown in Figures 1 and 2, respectively; an example of the identification of Type I, IIa, and IIb/d fibers on the basis of staining intensity is highlighted by the blue, red, and orange circles, respectively.
At $24 \mathrm{~h}$ : Fiber type proportions were similar for all groups; i.e., there was no effect of birth asphyxia or the presence of supplementary creatine on fiber type proportions (Figure 1e).

At $33 \mathrm{~d}$ : There was a significant difference in the effect of birth asphyxia on muscle fiber type proportions in male offspring from control-fed and creatine-supplemented dams $\left(\mathrm{p}_{\text {BIRTH }}<0.05\right)$. Male asphyxia offspring showed a significant decrease in the relative number of Types I and IIa fibers and a corresponding increase in IIb fibers $(P<0.05)$. These changes were prevented if their mother's diet had been supplemented with creatine during pregnancy $(P>0.05)$. Further, such changes were not present in female asphyxia offspring $(P>$ $0.05)$, and creatine alone had no effect $(P>0.05)$ on fiber types in the gastrocnemius muscle at $33 \mathrm{~d}$ of age in either sex (see Figure 2e).

\section{Muscle Fiber Cross-Sectional Area}

At $24 \mathrm{~h}$ : When examining the effect of birth asphyxia on muscle fiber CSA, we found significant changes in both male and female offspring $\left(\mathrm{p}_{\text {BIRTH }}<0.05\right)$. When compared to their c-section counterparts, the cross-sectional area (CSA) of all three muscle fiber types in asphyxia pups were significantly reduced at $24 \mathrm{~h}$ after birth in both males and females $(P<0.05)$. However, muscle fiber size in the gastrocnemius of animals born of mothers who consumed the creatine-supplemented diet during pregnancy, with or without birth asphyxia, were not different to $\mathrm{c}$-section animals $(P>0.05)$ (Figure $1 \mathrm{c}, \mathbf{d}$; Figure 3a). Additionally, these results suggested that oxidative fibers were most affected by birth asphyxia in both sexes, with a 41.4 and $53.5 \%$ reduction in the size of Type I fibers for males and females respectively, a 33.6 and $34.7 \%$ reduction in Type IIa, and a 32.1 and 33.8 reduction in Type IIb fibers.

At 33 d: A persistent effect of birth asphyxia on muscle fiber CSA was found in the gastrocnemius muscle at $33 \mathrm{~d}$ in both sexes $\left(\mathrm{p}_{\text {BIRTH }}<0.05\right)$. All three fiber types in the gastrocnemius of males, and Type I and IIb fibers in females, were significantly smaller than fibers taken from age-matched c-section animals (Figure 3c; $P<0.05$ ). Furthermore, fiber size in the creatine and $\mathrm{Cr}+$ asphyxia animals, in all fiber types, were not different to the c-section group (Figure 3c).

\section{Oxidative Capacity of Muscle Fibers}

At $24 \mathrm{~h}$ : Birth asphyxia significantly reduced oxidative capacity in Type I and IIa fibers in both male and female offspring at $24 \mathrm{~h}$ of age (Figure $3 \mathrm{~b} ; \mathrm{p}_{\text {BIRTH }}<0.05$ ). These changes were completely prevented when the maternal diet had been supplemented with creatine during pregnancy. Furthermore, creatine treatment alone caused no changes in oxidative capacity.

At 33 d: Male birth asphyxia animals showed a persistent reduction in oxidative capacity in all three fiber types compared to $c$-section males (Figure 3d; $\mathrm{p}_{\text {BIRTH }}<0.05$ ). No post-asphyxia effects were apparent in any fiber type in the gastrocnemius of female offspring at this age $(P>0.05)$. Maternal creatine treatment alone had no effect on succinate dehydrogenase (SDH) abundance in any fiber type of males or females at either age $(P$ $>0.05)$, but this antenatal treatment completely prevented all 


\section{Articles $\quad$ LaRosa et al.}

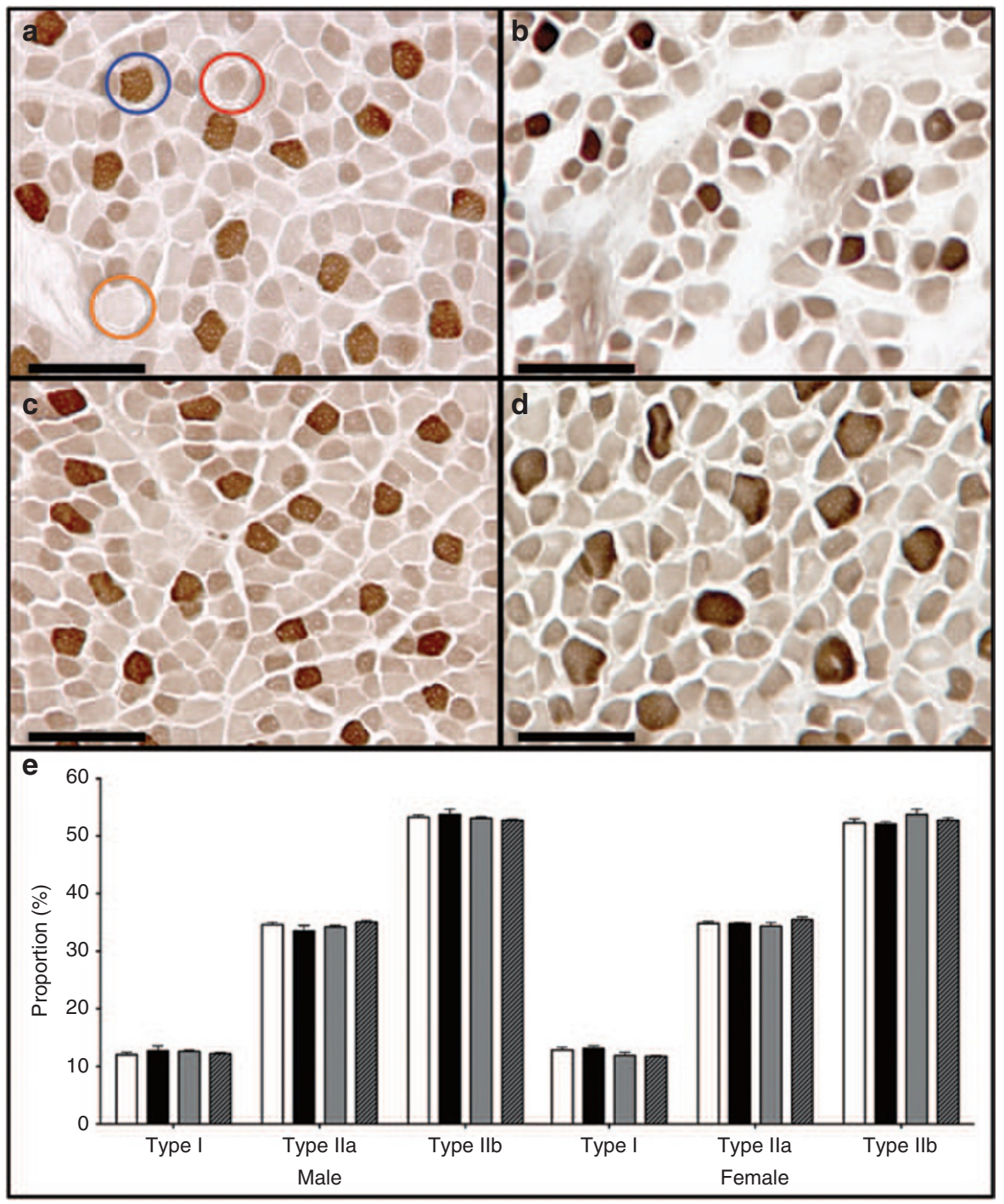

Figure 1. Histochemistry of the spiny mouse gastrocnemius muscle at $24 \mathrm{~h}$. Representative images of spiny mouse gastrocnemius muscle at $24 \mathrm{~h}$ of age stained for myofibrillar ATPase activity. Using this stain, Type I fibers stain dark (blue), Type lla fibers stain intermediate (red) and Type Ilb fibers remain pale (orange). (a) c-section, (b) asphyxia, (c) creatine, and (d) creatine + asphyxia. (e) Proportions of the different fiber types present in the $24 \mathrm{~h}$ gastrocnemius for the four treatment groups. C-section: white; asphyxia: black; creatine: gray; cr+asphyxia: gray hatched. Values are mean \pm SEM; $n=5$ males $/ 5$ females/group. Scale bar $=50 \mu \mathrm{m}$.

asphyxia-induced reductions in oxidative capacity in the male offspring.

\section{Functional Assessment of the Hindlimb}

Ex-vivo muscle function-24h group. Single twitch contractions induced by supra-maximal stimulation revealed that there were no significant differences between groups in peak twitch tension, time to peak twitch tension, or maximum tetanic force (Table 1). However, half relaxation time was significantly shorter in birth asphyxia pups compared to c-section controls for both males and females (Figure 4a; $\left.\mathrm{p}_{\text {BIRTH }}<0.05\right)$. Birth asphyxia was found to significantly affect resistance to fatigue in the gastrocnemius muscle at $24 \mathrm{~h}$ or age $\left(\mathrm{p}_{\text {BIRTH }}<0.05\right)$. Assessed from the reduction in force production during repeated tetanic contractions, fatigue was greater in the birth asphyxia offspring after 50 contractions compared to the c-section offspring $(P<0.05)$ for both males and females (Figure 4b). However, at the 300th train of contraction, there was no significant difference between groups (Table 1). Maternal creatine treatment alone had no effect on any contractile parameters. However, the decrease of half relaxation time and the increased in fatigue were not present after birth asphyxia in pups from creatine-treated mothers $(P$ $>0.05$; Figure $4 \mathrm{~b}$ ).

\section{Rotarod Test}

Fatigue and endurance, assessed by Rotarod performance at postnatal days 14 and 21, were found to be persistently reduced by birth asphyxia $\left(\mathrm{p}_{\text {BIRTH }}<0.05\right)$. At postnatal day 14 , male but not female birth asphyxia pups had a significantly shorter latency to fall than their c-section counterparts $(P<$ 0.05), as shown in Figure 5a. This deficit was still evident at 


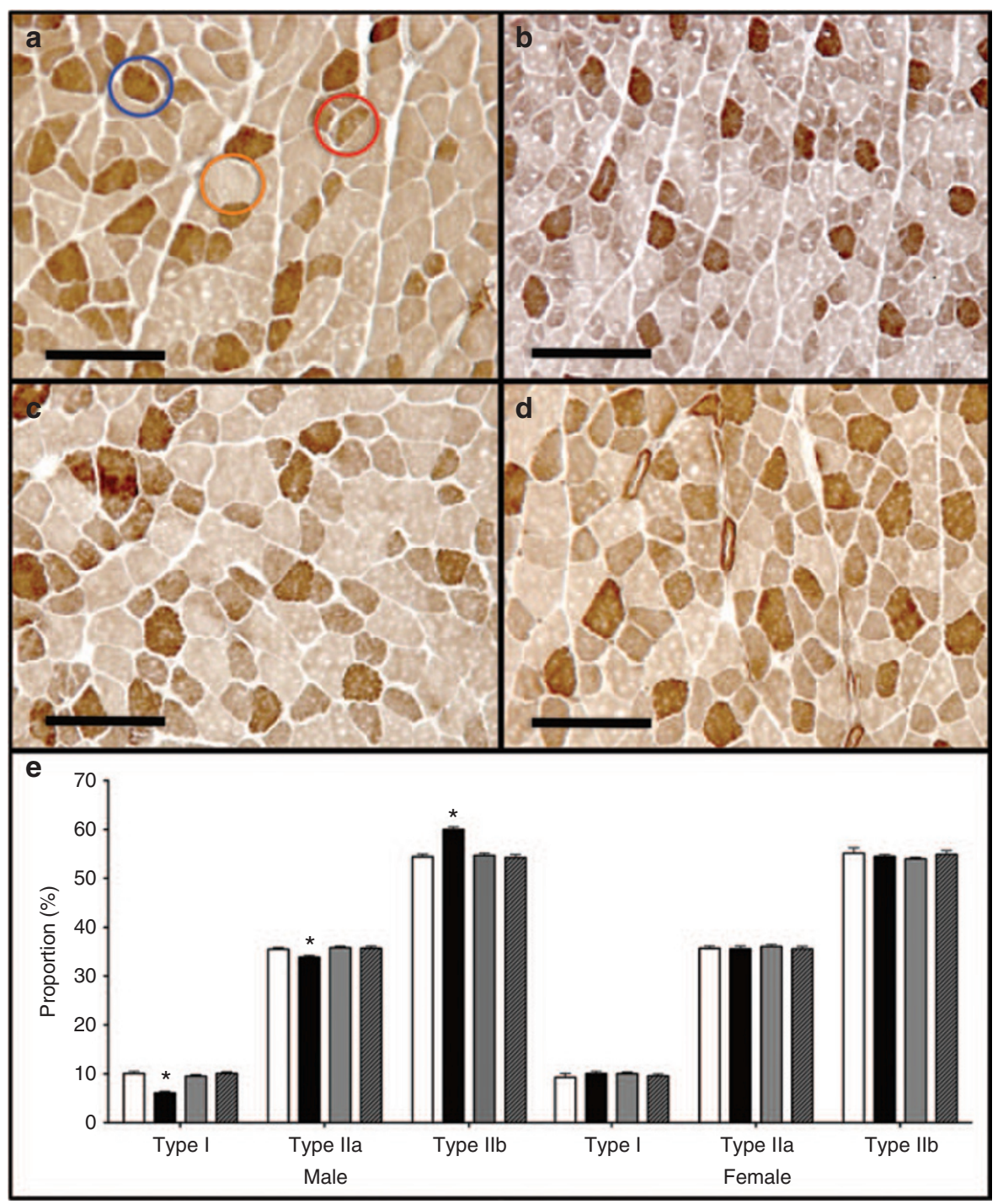

Figure 2. Histochemistry of the spiny mouse gastrocnemius muscle at $33 \mathrm{~d}$. Representative images of ATPase stained spiny mouse gastrocnemius at $33 \mathrm{~d}$ of age. Type I fibers stain dark (blue), Type lla fibers stain intermediate (red) and Type llb fibers remain pale (orange). (a) c-section, (b) asphyxia, (c) creatine, and (d) creatine + asphyxia. (e) Proportions of the different fiber types present in the gastrocnemius at $33 \mathrm{~d}$ of age for the four treatment groups. C-section: white; asphyxia: black; creatine: gray; $\mathrm{cr}+$ asphyxia: gray hatched. Values are mean $\pm \mathrm{SEM} ; n=5$ males $/ 5$ females/group. * indicates significant difference to all other groups $(P<0.05)$. Scale bar $=100 \mu \mathrm{m}$.

postnatal day 21 (Figure $5 \mathbf{b}$ ). The performance of animals supplemented with creatine during pregnancy, regardless of sex or whether they underwent asphyxia at birth, did not differ from the $\mathrm{c}$-section offspring at either postnatal age $(P>0.05)$.

\section{DISCUSSION}

This study shows that birth asphyxia disrupts the structure of the gastrocnemius muscle, a major, mixed fiber type axial skeletal muscle, and some of these changes-in particular, fiber size and oxidative capacity-persist until at least $33 \mathrm{~d}$ of age in this precocial species. The effects of birth asphyxia on the skeletal muscle appear to be greater in males compared to females. There was also a significant decrease in the relative number of "slow" (Type I) and increase in the "fast" Type IIb fibers in male offspring at $33 \mathrm{~d}$ of age, which may be the structural counterpart to the reduction in functional endurance observed in the ex-vivo muscle studies at $24 \mathrm{~h}$ of age. Furthermore, the male birth-asphyxia offspring showed a functional limitation, in that they were less able to cope with the accelerating Rotarod for significantly less time than their c-section counterparts. Finally, we show that all of the early and long-term postnatal changes induced by birth asphyxia were prevented when the mother had received a creatine-supplemented diet for the second half of pregnancy.

Our findings on the impact of birth asphyxia on muscle fiber size and the relative number of oxidative and glycolytic fibers is consistent with the changes noted for skeletal muscle in children with $\mathrm{CP}$, where reduced muscle fiber size and alterations in fiber type proportions have been reported $(11,18,20)$. These changes may be the result of hypoxia-related upregulation of protease activity and expression of proatrophic genes such as atrogin-1 and MuRF1 $(7,9,12)$, which we have 

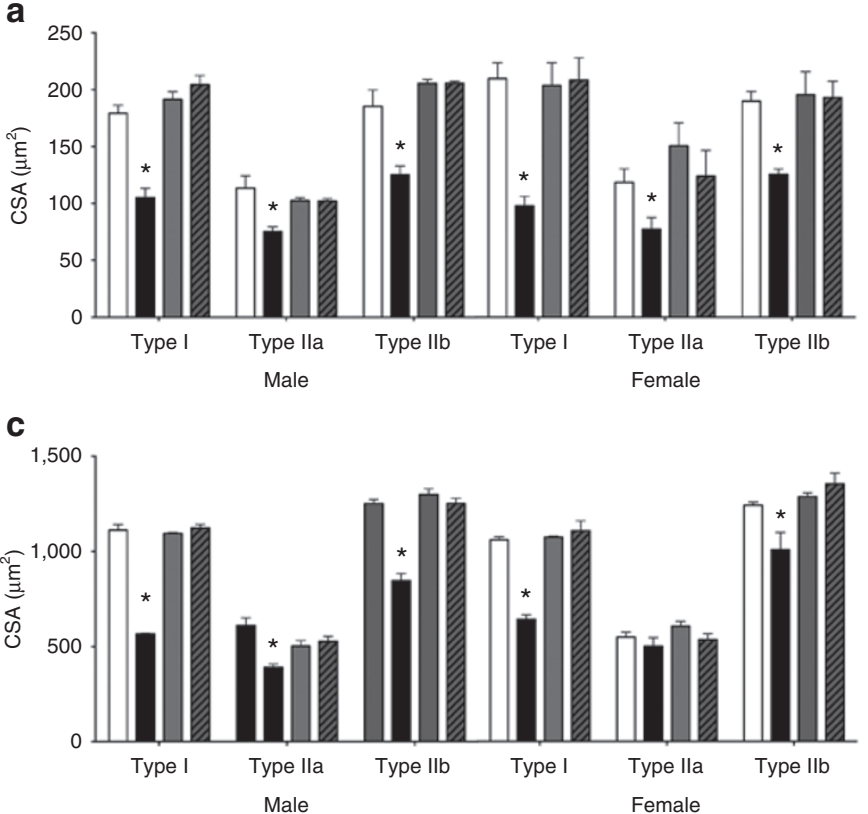

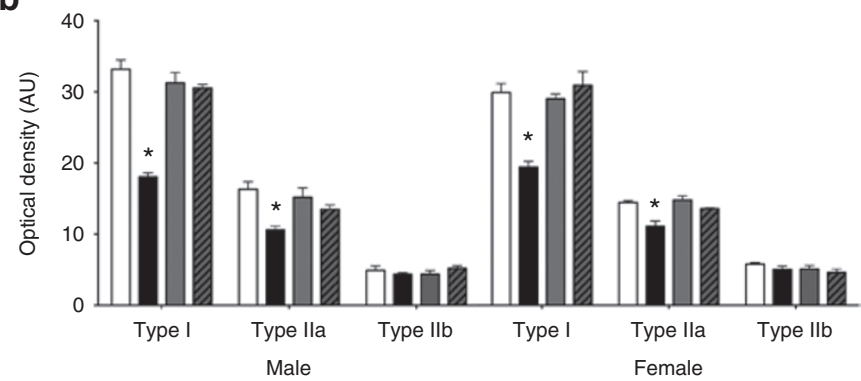

d

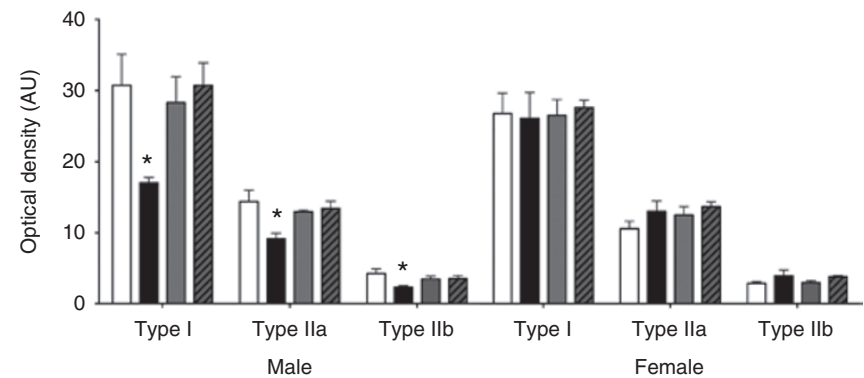

Figure 3. Cross-sectional area (CSA) and oxidative capacity of different muscle fiber types in the spiny mouse gastrocnemius muscle. (a) Mean CSA and (b) succinate dehydrogenase (SDH) abundance of all fiber types in the spiny mouse gastrocnemius at $24 \mathrm{~h}$ of age for all treatment groups. All fiber types were significantly smaller in asphyxia pups than all other groups $(P<0.05)$. Asphyxia reduced SDH abundance in Type I and Ila fibers compared to all other groups $(P<0.05)$. (c) CSA and (d) SDH abundance of all fiber types in the spiny mouse gastrocnemius at $33 \mathrm{~d}$ of age for the four treatment groups. All fiber types from asphyxia males, and Type I and IIb fibers in females, were persistently smaller than all other groups $(P<0.05)$. SDH abundance was persistently reduced in asphyxia males compared to all other groups $(P<0.05)$. C-section: white; asphyxia: black; creatine: gray; cr+asphyxia: gray hatched. Values are mean $\pm \mathrm{SEM} ; n=$ males $/ 5$ females/group. * indicates significant difference to all other groups $(P<0.05)$.

Table 1. Ex-vivo muscle function of $24 \mathrm{~h}$ gastrocnemius muscles.

\begin{tabular}{|c|c|c|c|c|c|}
\hline & & C-section & Asphyxia & Creatine & $\mathrm{Cr}+\mathrm{Asph}$ \\
\hline Peak twitch tension $\left(\mathrm{N} / \mathrm{cm}^{2}\right)$ & Female & $9.63 \pm 0.85$ & $11.03 \pm 2.75$ & $10.17 \pm 2.05$ & $12.15 \pm 1.98$ \\
\hline Time to peak twitch tension (seconds) & Female & $0.02 \pm 0.003$ & $0.02 \pm 0.001$ & $0.03 \pm 0.003$ & $0.02 \pm 0.003$ \\
\hline Max tetanic force $\left(\mathrm{N} / \mathrm{cm}^{2}\right)$ & Male & $19.50 \pm 3.70$ & $23.69 \pm 3.08$ & $24.55 \pm 5.97$ & $22.98 \pm 6.25$ \\
\hline \multirow[t]{2}{*}{ Fatigue index 2 (300th train) } & Male & $0.24 \pm 0.03$ & $0.23 \pm 0.02$ & $0.21 \pm 0.05$ & $0.24 \pm 0.03$ \\
\hline & Female & $0.24 \pm 0.03$ & $0.23 \pm 0.01$ & $0.032 \pm 0.09$ & $0.28 \pm 0.04$ \\
\hline \multirow[t]{2}{*}{ Muscle weight (mg) } & Male & $0.029 \pm 0.006$ & $0.028 \pm 0.004$ & $0.024 \pm 0.004$ & $0.024 \pm 0.005$ \\
\hline & Female & $0.026 \pm 0.003$ & $0.027 \pm 0.005$ & $0.022 \pm 0.002$ & $0.029 \pm 0.003$ \\
\hline Muscle weight:body weight & Male & $0.188 \pm 0.047$ & $0.196 \pm 0.035$ & $0.226 \pm 0.033$ & $0.219 \pm 0.035$ \\
\hline
\end{tabular}

No effect of birth or diet was observed for any of the parameters $(P>0.05)$. Values are means \pm SEM. $n=5 / g r o u p$. Statistical analysis, tw-way ANOVA.

previously reported were upregulated in the diaphragm of the spiny mouse at $24 \mathrm{~h}$ after birth asphyxia $(13,14)$. Additionally, expression of myostatin, another proatrophic gene, has been reported to be increased in the muscles of children with $\mathrm{CP}$ $(14,25)$. Therefore, there may be disruption in the balance between protein synthesis and degradation to favor the latter, resulting in decreased muscle fiber size. Birth asphyxia may be acting not only through proteolytic pathways to damage the muscle and decrease their size, but also by interrupting the normal growth of the muscle, leaving them smaller in adulthood. Finkelstein et al. reported that, in the first days of life, the CSA of all three fiber types significantly increased in various muscles of the sheep hindlimb $(15,26)$. Furthermore, CSA continued to increase exponentially over postnatal life until reaching a maximum at adulthood.

In the present study, we found that development to $33 \mathrm{~d}$ of age was associated with a reduction in the relative number of Type I and IIa fibers, with a corresponding increase in Type 
a

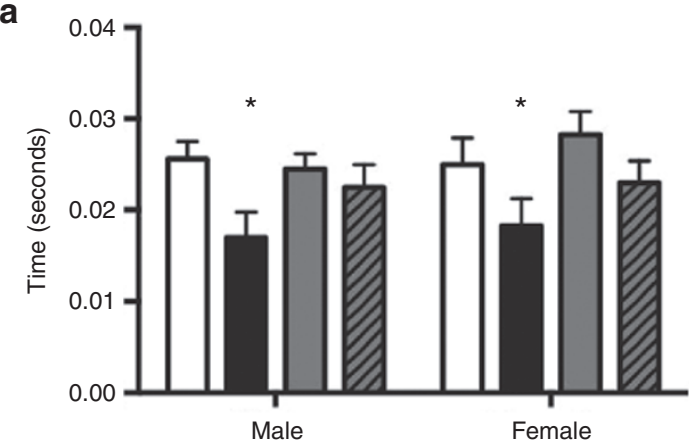

b

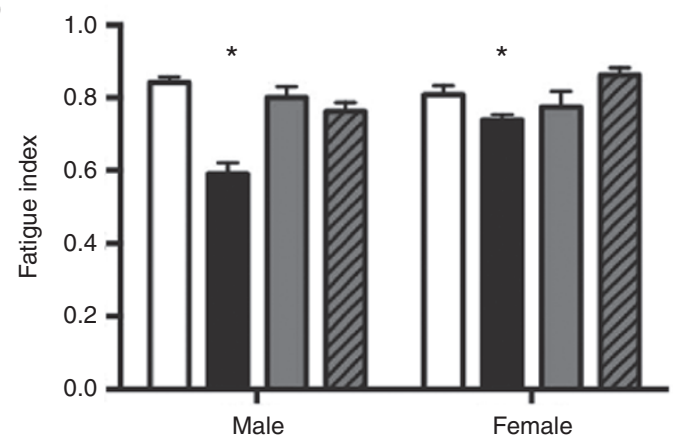

Figure 4. Ex-vivo Muscle function of the spiny mouse gastrocnemius muscle at $24 \mathrm{~h}$ of age. (a) Mean time to half relaxation tension values obtained for our four treatment groups. (b) Mean reduction in force production at the 50th contraction of a fatigue train experiment, expressed as a fraction of the force at the 1st contraction. C-section: white; asphyxia: black; creatine: gray; $\mathrm{cr}+$ asphyxia: gray hatched. Values are mean $\pm \mathrm{SEM}$; $n=$ males $/ 5$ females/group. ${ }^{*}$ indicates significant difference to all other groups $(P<0.05)$.

IIb fibers in male offspring who underwent birth asphyxia compared to their c-section counterparts. This change was not evident at $24 \mathrm{~h}$ after birth, suggesting that it might develop progressively, and since it was not present in female offspring, this alteration in fiber proportions may therefore be affected by the different hormonal profiles of males and females during development. An increase in ROS and RNS production in response to birth asphyxia could also interrupt aerobic metabolism, causing damage to the mitochondria $(16,27)$, possibly contributing to the increased fatigue at $24 \mathrm{~h}$. While we cannot rule out changes in muscle innervation and neural activity, both of which are well known to influence muscle fiber phenotype $(17,18,21,28)$, we observed no obvious difference in the behavior of male and female offspring that suggests such a fundamental change of muscle activity.

However, the Rotarod test revealed a deficit in motor performance in the male offspring, which may be a consequence of this sex-dependent effect of birth asphyxia on muscle fiber types. Fewer Type I and IIa fibers and increased numbers of Type IIb fibers, along with the decrease in oxidative capacity and thus fatigue resistance (determined from a decreased $\mathrm{SDH}$ abundance), are likely to result in an overall reduction in oxidative potential, and therefore a lower resistance to fatigue. Indeed, in a related study, we have reported a significant reduction in fatigue resistance in the diaphragm of the same animals when they were tested ex-vivo at $33 \mathrm{~d}(15,19)$. Furthermore, the significant effect of birth asphyxia on oxidative fibers (Type I and Type IIa) may have particular importance for human infants as the human gastrocnemius muscle is comprised of almost 53\% Type I fibers (29) as well as for other muscles with high proportions of Type I fibers (e.g., soleus muscle), which have a crucial role in the maintenance of posture. It is not unreasonable to assume that other muscles in the hindlimb, or of the upper limbs and trunk, may be similarly affected by birth asphyxia. If Type I fibers in these muscles are also compromised structurally and functionally, resulting in functional reductions in fatigue resistance, this could be contributing to the postural and gait abnormalities $(17,18)$, and the muscle weakness observed in CP children (30). Indeed a recent study found that after a walking trial to induce muscle fatigue, adolescents with CP exhibited significantly reduced postural control in comparison to age-matched controls (31).

After birth asphyxia, female offspring performed similarly on the Rotarod to controls at both postnatal time points tested, which was in contrast to the males. This could be related to the females having a similar fiber type proportion and oxidative capacity to c-section animals, despite the similar reduction in CSA. The mechanisms involved in this sexual dimorphism are unknown, however, females are known to be able to repair exercise induced muscle damage better than males (32), which has been related to the anti-inflammatory effects of estrogen (33). Furthermore, the estrogen receptor is present in the spiny mouse ovary from postnatal day 7 (34). Therefore, it is possible that the female endocrine environment could be contributing to either the protection of axial skeletal muscle following birth asphyxia, or their recovery post-asphyxia over time after asphyxia.

The major limitation to effective clinical management of birth asphyxia is the inability to predict or detect its occurrence. Birth asphyxia is essentially a global fetal/perinatal insult involving severe hypoxia and hypercapnia, leading to acidemia and widespread energy failure in tissues due to impaired mitochondrial regeneration of ATP. Hence, we have used creatine supplementation as a prophylactic therapy, and previously shown that it is effective in preventing asphyxiainduced damage to the neonatal brain (23), kidney (24), and diaphragm $(14,15)$ in this species. Here, we show similar protective benefits in the gastrocnemius muscle. A likely explanation of the protective effect of creatine treatment is that it slows down the rate of ATP depletion in tissues where it is most likely to occur because of a high dependence on ATP-related cellular processes. Creatine is also known to attenuate the accumulation of ROS and to produce vasodilatation, effects that might reduce oxidative stress in muscle following the hypoxia and acidemia caused by the birth asphyxia protocol. Furthermore, this study revealed that supplementing the maternal diet with creatine during pregnancy did not result in any significant changes in normal skeletal muscle structure or function (creatine group), either in the immediate neonatal period or later in life. These results, taken together with our previous work on the brain (23), diaphragm $(14,15)$, and kidney (24), confirm 


\section{Articles LaRosa et al.}

a

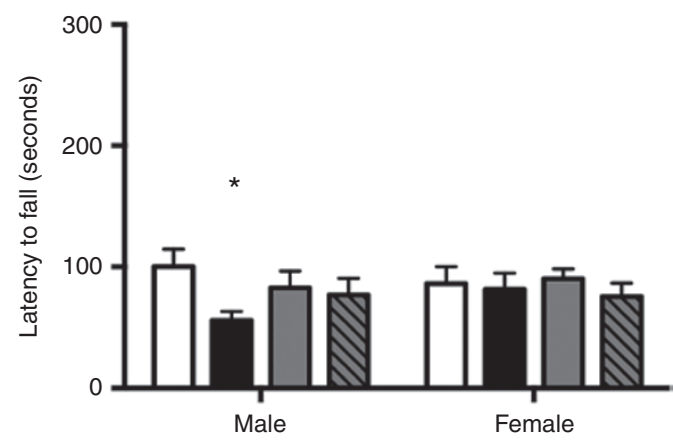

b

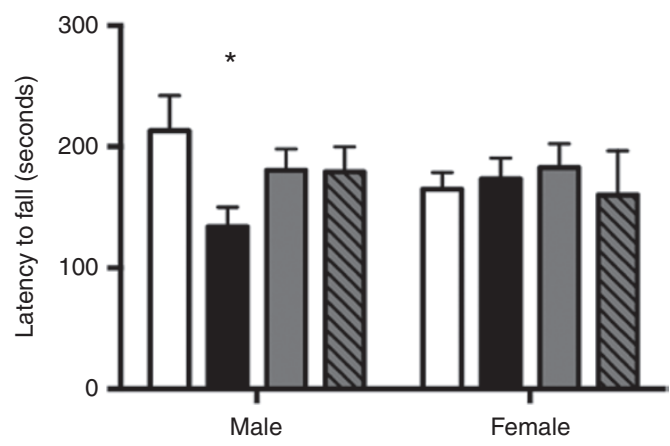

Figure 5. Rotarod performance of the spiny mouse. Summary of mean latency to fall from the Rotarod apparatus over five trials at postnatal days 14 (a) and 21 (b) at day 21. C-section: white; asphyxia: black; creatine: gray; $\mathrm{cr}+$ asphyxia: gray hatched. Values are mean $\pm \mathrm{SEM} ; n=$ males $/ 5$ females/ group. * indicates significant difference to all other group $(P<0.05)$.

that maternal dietary creatine supplementation during pregnancy, could be a safe and readily available prophylactic treatment for the prevention of birth asphyxia-induced mortality and morbidity.

\section{METHODS}

\section{Ethics and Animal Husbandry}

All experiments had prior approval from Monash University Animal Ethics Committee and the Australian Government Department of Primary Industries, and were conducted in accordance with the Australian Code of Practice for the Care and Use of Animals for Scientific Purposes. Spiny mice used for this study were obtained from our own laboratory colony and housed, bred and time-mated as previously described (35).

\section{Diet}

Pregnant dams were fed either a control diet of standard rat and mouse chow (2.16mg creatine/g) throughout pregnancy, or chow supplemented with $5 \%$ creatine monohydrate $(32.44 \mathrm{mg}$ creatine/g; Specialty Feeds, Glen Forrest, Perth, Australia; Creatine, Sigma) from day 20 of gestation to term (day 39 ). Water was freely available and animals were housed in family groups.

\section{Experimental Groups}

Four groups of animals were studied-those delivered by cesarean section from mothers fed the control diet (c-section) or creatine diet (creatine), and those born after a period of in-utero hypoxia-ischemia (asphyxia, $\mathrm{Cr}+$ asphyxia).

\section{Birth Asphyxia}

The model of birth asphyxia used in this study has been extensively described and characterized by us $(14,15,23,24,36)$. Briefly, on day 38 of gestation, the dam was killed by cervical dislocation and the isolated pregnant uterus placed in saline $(0.9 \%)$ at $37^{\circ} \mathrm{C}$ for $7.5-8 \mathrm{~min}$. Fetuses were expelled and resuscitated by gentle palpation of the chest. Control pups were delivered immediately by cesarean section. Pups were crossfostered to a lactating dam who had been maintained on a normal diet throughout pregnancy and had given birth within the previous $24 \mathrm{~h}$ (the dam's own litter was removed to ensure adequate lactation for the cross-foster pups). Pups from all the treatment groups (c-section, asphyxia, creatine, $\mathrm{Cr}+$ asphyxia) were then assigned to one of two subgroups where they were either killed by decapitation at the beginning of postnatal day 2 (i.e., $24 \mathrm{~h}$ after birth), or were nursed by the cross-foster dam until $33 \pm 2 \mathrm{~d}$ when they were killed by cervical dislocation.

\section{Tissue Collection}

At postmortem, both hindlimbs were immediately removed, skinned and placed in ice-cold, Krebs solution $(118 \mathrm{mmol} / \mathrm{l} \mathrm{NaCl}, 4.7 \mathrm{mmol} / \mathrm{l}$ $\mathrm{KCl}, 1.2 \mathrm{mmol} / \mathrm{l} \mathrm{MgSO}, 1.2 \mathrm{mmol} / \mathrm{K} \mathrm{KH}_{2} \mathrm{PO}_{4}, 2.5 \mathrm{mmol} / \mathrm{l} \mathrm{CaCl}$, $25 \mathrm{mmol} / \mathrm{l} \mathrm{NaHCO}_{3}$, and $11.1 \mathrm{mmol} / \mathrm{l}$ glucose), which had been prebubbled with carbogen $\left(5 \% \mathrm{CO}_{2}, 95 \% \mathrm{O}_{2}\right.$. For the pups killed at $24 \mathrm{~h}$ of age ( $n=5$ males/5 females/group), the left gastrocnemius muscle was used for ex vivo functional assessment (see below), and the right gastrocnemius muscle was snap frozen under resting tension in precooled isopentane and stored at $-80^{\circ} \mathrm{C}$ for histochemical analyses. At $33 \mathrm{~d}$ of age, both right and left gastrocnemius muscles were snap frozen in isopentane ( $n=5$ males $/ 5$ females/group).

\section{Muscle Total Creatine Content at $24 \mathrm{~h}$}

The level of creatine loading in the gastrocnemius $24 \mathrm{~h}$ after birth, measured as concentration of total creatine (creatine + phosphocreatine), was determined in cesarean-delivered pups from control and creatinefed mothers ( $n=5$ for both groups) as previously published $(36,37)$.

\section{Histochemistry of Gastrocnemius Samples}

Myofibrillar ATPase. Transverse sections $(10 \mu \mathrm{m})$ were cut from the widest point of the frozen gastrocnemius muscle using a cryostat microtome (Leica) and adhered to Superfrost Plus slides (Menzel-Gläser, Thermo Scientific, Waltham, MA). Myofibrillar ATPase staining was used to differentiate Type I (slow-oxidative), Type IIa (fast-oxidative), and Type IIb/IId (fast-glycolytic) fibers as previously described $(14,15,38)$, with the following modifications: sections were preincubated in acetate buffer for $7 \mathrm{~min}(\mathrm{pH}$ 4.3), then incubated for $15 \mathrm{~min}$ in ATPase solution ( $\mathrm{pH} 9.4$ ) at $37^{\circ} \mathrm{C}$. Using this protocol, type I fibers stain dark, type IIa stain intermediate, and type IIb/IId stain pale. Using these stained sections, the overall proportions of the different fiber types present in the gastrocnemius, as well as the CSA of a minimum of 20 individual fibers per type, per animal were determined.

\section{Oxidative enzyme activity.}

$\mathrm{SDH}$ abundance was used to determine the oxidative capacity of individual gastrocnemius fibers, using adjacent sections to those used for ATPase analysis. The SDH protocol was modified from a previously described method $(15,39)$. Briefly, sections were incubated in 100 $\mathrm{mmol} / \mathrm{l}$ phosphate buffer $(\mathrm{pH} 7.6), 50 \mathrm{mmol} / \mathrm{l}$ succinate, $5 \mathrm{mmol} / \mathrm{l}$ Ethylenediaminetetraacetic acid (EDTA), $1.5 \mathrm{mmol} / \mathrm{l}$ nitro-blue tetrazolium, $1 \mathrm{mmol} / \mathrm{l}$ sodium azide, and $0.2 \mathrm{mmol} / \mathrm{l}$ phenazine at 37 ${ }^{\circ} \mathrm{C}$ for $15 \mathrm{~min}$. Sections were then submerged sequentially in 30,60 , and $90 \%$ aqueous acetone before being rinsed in $\mathrm{dH}_{2} \mathrm{O}$. Incubation without succinate was carried out to determine nonspecific staining. Densitometric analysis using Image J software (NIH, MA) was then employed to categorize the oxidative capacity of individual fibers based on their staining intensity, with greater optical density indicating higher abundance of this enzyme $(15,37)$. As these were serial sections to those stained for ATPase activity, the ATPase-stained images were used as a reference and the same fibers were found on the SDHstained slides. This allowed the mean oxidative capacity for each fiber type to be determined for each animal.

\section{Functional Assessment of Hindlimb Muscle}

Ex-vivo hindlimb muscle function at $24 \mathrm{~h}$. As it was not possible to assess motor performance at $24 \mathrm{~h}$ of age using the Rotarod, due to their age and limited mobility, we utilized ex vivo muscle function testing to functionally assess the gastrocnemius muscle in this group. To isolate this muscle from the lower leg, fascia on either side of the muscle was carefully removed and forceps were inserted beneath the Achilles tendon. The proximal end of the muscle remained attached to the knee joint, and the entire muscle was suspended in an organ 
bath, with the knee joint fixed to a tissue holder and the Achilles tendon attached to a force transducer using surgical silk. The organ bath was filled with Krebs solution, maintained at $34^{\circ} \mathrm{C}$ and bubbled with carbogen. During the initial equilibration period, the muscle was adjusted to the optimum length for the production of maximal twitch force. Muscle contractions were induced by electrical field stimulation using a pair of electrodes placed on either side of the muscle, connected to a stimulator (Grass S88). Stimuli ( $0.2 \mathrm{~ms}$ duration) to induce single twitch contractions were delivered at 1 pulse per second at $1.2-1.5 \times$ the stimulus strength needed to obtain maximum twitch contraction. Tetanic contractions were obtain using a train of pulses (pulse duration $0.2 \mathrm{~ms}$, at $40 \mathrm{~Hz}, 330 \mathrm{~ms}$ train duration), and fatigue was assessed using this tetanic stimulation repeated at 1 train/second for $300 \mathrm{~s}$ ( $n=5 \mathrm{males} / 5$ females/group). All twitch tension and fatigue recordings were stored digitally using an A-D converter, and analyzed later using Labchart software (AD Instruments, Sydney, Australia).

Rotarod assessment. A Rotarod test was conducted on postnatal days 14 and 21 to assess motor co-ordination and endurance as previously described (40). A standard accelerating Rotarod apparatus was modified by adding 12 plastic rungs, placed $1.5 \mathrm{~cm}$ above the surface of the inner axle. The addition of the rungs made it a requirement that the animal correctly place all four paws on the rungs to stay on the apparatus as it rotated. This addition was made to increase the difficulty of the Rotarod test for the spiny mice, which are more agile compared to altricial laboratory rodents. Once started, the speed increased by 7.2 revolutions $/ \mathrm{min} / \mathrm{min}$. All animals were allowed two learning trials on the Rotarod, and then the latency to fall was determined for five trials and a maximum latency of $5 \mathrm{~min}$ was allowed for each trial. The mean latency over the five trials was then determined.

\section{Statistical Analysis}

All data are presented as the mean \pm SEM. Data were assessed by threeway ANOVA, to establish the effects of mode of birth $\left(\mathrm{p}_{\text {BIRTH }}\right)$, maternal $\operatorname{diet}\left(\mathrm{p}_{\mathrm{DIET}}\right)$, and sex $\left(\mathrm{p}_{\mathrm{SEX}}\right)$ of the offspring. Sex of the offspring significantly interacted with the effects of birth asphyxia; therefore for clarity, data has been presented split for sex, with male and female offspring assessed independently using a two-way ANOVA, assessing the effects of birth asphyxia $\left(\mathrm{p}_{\mathrm{BIRTH}}\right)$ and maternal diet $\left(\mathrm{p}_{\mathrm{DIET}}\right)$. Post hoc analysis with Tukey's multiple comparisons was conducted using statistical software (Prism 6, Graphpad software) (15). Numbers $(n)$ indicate the number of pups, each obtained from a separate litter, and also represent the number of dams in each group. Statistical significance was set at $P<0.05$.

\section{ACKNOWLEDGMENTS}

The authors thank Samir Mesanovic for completing the creatine assay, and Udani Ratnayake, Tracey Quinn, Lisa Davis, and Annike Griffey for conducting the Rotarod assessment.

\section{AUTHOR CONTRIBUTIONS}

D.A.L. conducted all animal experiments, tissue collections, experimental analysis, data interpretation, and was the principle writer of the manuscript. S.J.E. assisted in animal generation. R.J.S. and D.W.W. contributed experimental design and data interpretation. H.D. assisted in experimental design and funded the study. All participated in the drafting of this manuscript and approved the final manuscript.

\section{STATEMENT OF FINANCIAL SUPPORT}

The authors acknowledge receipt of the following financial support for the research and authorship of this article: Project grant funding from the NH\&MRC and the Cerebral Palsy alliance, to H.D. H.D. was supported by NH\&MRC fellowships, and the Victorian Government's Operational Infrastructure Support Program also partially supported this work. D.A.L. and S.J.E. were supported by Australian Postgraduate Award scholarships when these experiments were completed. The authors have no ties to products in this study or any conflicts to disclose.

Disclosure: None.

\section{REFERENCES}

1. Lawn JE, Cousens S, Zupan J; Lancet Neonatal Survival Steering Team. 4 million neonatal deaths: when? Where? Why? Lancet 2005;365:891-900.
2. Kurinczuk JJ, White-Koning M, Badawi N. Epidemiology of neonatal encephalopathy and hypoxic-ischaemic encephalopathy. Early Hum Dev 2010;86:329-38.

3. Nordström L, Arulkumaran S. Intrapartum fetal hypoxia and biochemical markers: a review. Obstet Gynecol Surv 1998;53:645-57.

4. Campbell AG, Dawes GS, Fishman AP, Hyman AI. Regional redistribution of blood flow in the mature fetal lamb. Circ Res 1967;21:229-35.

5. Caquelard F, Burnet H, Tagliarini F, Cauchy E, Richalet JP, Jammes Y. Effects of prolonged hypobaric hypoxia on human skeletal muscle function and electromyographic events. Clin Sci (Lond) 2000;98:329-37.

6. Allen DG, Lamb GD, Westerblad H. Skeletal muscle fatigue: cellular mechanisms. Physiol Rev 2008;88:287-332.

7. Caron MA, Thériault ME, Paré ME, Maltais F, Debigaré R. Hypoxia alters contractile protein homeostasis in L6 myotubes. FEBS Lett 2009;583: 1528-34.

8. Simpson JA, van Eyk JE, Iscoe S. Hypoxemia-induced modification of troponin I and T in canine diaphragm. J Appl Physiol (1985) 2000;88:753-60.

9. Russell AP. Molecular regulation of skeletal muscle mass. Clin Exp Pharmacol Physiol 2010;37:378-84.

10. Mohanraj P, Merola AJ, Wright VP, Clanton TL. Antioxidants protect rat diaphragmatic muscle function under hypoxic conditions. J Appl Physiol (1985) 1998;84:1960-6.

11. Hoppeler H, Kleinert E, Schlegel C, et al. Morphological adaptations of human skeletal muscle to chronic hypoxia. Int J Sports Med 1990;11 Suppl 1:S3-9.

12. Weiser MR, Williams JP, Moore FD Jr, et al. Reperfusion injury of ischemic skeletal muscle is mediated by natural antibody and complement. J Exp Med 1996;183:2343-8.

13. Clanton TL, Zuo L, Klawitter P. Oxidants and skeletal muscle function: physiologic and pathophysiologic implications. Proc Soc Exp Biol Med 1999;222:253-62.

14. Cannata DJ, Ireland Z, Dickinson $\mathrm{H}$, et al. Maternal creatine supplementation from mid-pregnancy protects the diaphragm of the newborn spiny mouse from intrapartum hypoxia-induced damage. Pediatr Res 2010;68:393-8.

15. LaRosa DA, Ellery SJ, Parkington HC, Snow RJ, Walker DW, Dickinson H. Maternal creatine supplementation during pregnancy prevents long-term changes in diaphragm muscle structure and function after birth asphyxia. PLoS One 2016;11:e0149840.

16. du Plessis AJ, Volpe JJ. Perinatal brain injury in the preterm and term newborn. Curr Opin Neurol 2002;15:151-7.

17. Graham HK, Aoki KR, Autti-Rämö I, et al. Recommendations for the use of botulinum toxin type A in the management of cerebral palsy. Gait Posture 2000;11:67-79.

18. Rose J, Haskell WL, Gamble JG, Hamilton RL, Brown DA, Rinsky L. Muscle pathology and clinical measures of disability in children with cerebral palsy. J Orthop Res 1994;12:758-68.

19. Kerr Graham H, Selber P. Musculoskeletal aspects of cerebral palsy. J Bone Joint Surg Br 2003;85:157-66.

20. Malaiya R, McNee AE, Fry NR, Eve LC, Gough M, Shortland AP. The morphology of the medial gastrocnemius in typically developing children and children with spastic hemiplegic cerebral palsy. J Electromyogr Kinesiol 2007;17:657-63.

21. Matsakas A, Patel K. Skeletal muscle fibre plasticity in response to selected environmental and physiological stimuli. Histol Histopathol 2009;24: 611-29.

22. Love SC, Novak I, Kentish M, et al.; Cerebral Palsy Institute. Botulinum toxin assessment, intervention and after-care for lower limb spasticity in children with cerebral palsy: international consensus statement. Eur J Neurol 2010;17 Suppl 2:9-37.

23. Ireland Z, Castillo-Melendez M, Dickinson H, Snow R, Walker DW. A maternal diet supplemented with creatine from mid-pregnancy protects the newborn spiny mouse brain from birth hypoxia. Neuroscience 2011;194:372-9.

24. Ellery SJ, Ireland Z, Kett MM, Snow R, Walker DW, Dickinson H. Creatine pretreatment prevents birth asphyxia-induced injury of the newborn spiny mouse kidney. Pediatr Res 2013;73:201-8. 


\section{Articles | LaRosa et al.}

25. Smith LR, Pontén E, Hedström Y, et al. Novel transcriptional profile in wrist muscles from cerebral palsy patients. BMC Med Genomics 2009;2:44.

26. Finkelstein DI, Andrianakis P, Luff AR, Walker DW. Developmental changes in hindlimb muscles and diaphragm of sheep. Am J Physiol 1992;263(4 Pt 2):R900-8.

27. Blomgren K, Hagberg H. Free radicals, mitochondria, and hypoxiaischemia in the developing brain. Free Radic Biol Med 2006;40:388-97.

28. Walker DW, Luff AR. Functional development of fetal limb muscles: a review of the roles of activity, nerves and hormones. Reprod Fertil Dev 1995;7:391-8.

29. Edgerton VR, Smith JL, Simpson DR. Muscle fibre type populations of human leg muscles. Histochem J 1975;7:259-66.

30. Stackhouse SK, Binder-Macleod SA, Lee SC. Voluntary muscle activation, contractile properties, and fatigability in children with and without cerebral palsy. Muscle Nerve 2005;31:594-601.

31. Vitiello D, Pochon L, Malatesta D, Girard O, Newman CJ, Degache F. Walking-induced muscle fatigue impairs postural control in adolescents with unilateral spastic cerebral palsy. Res Dev Disabil 2016;53-54:11-8.

32. Komulainen J, Koskinen SO, Kalliokoski R, Takala TE, Vihko V. Gender differences in skeletal muscle fibre damage after eccentrically biased downhill running in rats. Acta Physiol Scand 1999;165:57-63.

33. Tiidus PM. Estrogen and gender effects on muscle damage, inflammation, and oxidative stress. Can J Appl Physiol 2000;25:274-87.
34. Hułas-Stasiak M, Gawron A. Immunohistochemical localization of estrogen receptors ERalpha and ERbeta in the spiny mouse (Acomys cahirinus) ovary during postnatal development. J Mol Histol 2007;38: $25-32$.

35. Dickinson H, Walker DW, Cullen-McEwen L, Wintour EM, Moritz K. The spiny mouse (Acomys cahirinus) completes nephrogenesis before birth. Am J Physiol Renal Physiol 2005;289:F273-9.

36. Ireland Z, Dickinson H, Snow R, Walker DW. Maternal creatine: does it reach the fetus and improve survival after an acute hypoxic episode in the spiny mouse (Acomys cahirinus)? Am J Obstet Gynecol 2008;198:431. e1-6.

37. LaRosa DA, Cannata DJ, Arnould JPY, O'Sullivan LA, Snow RJ, West JM. Changes in muscle composition during the development of diving ability in the Australian fur seal. Aust J Zool 2012;60:81.

38. Brooke MH, Kaiser KK. Some comments on the histochemical characterization of muscle adenosine triphosphatase. J Histochem Cytochem 1969;17:431-2.

39. Pette D, Tyler KR. Response of succinate dehydrogenase activity in fibres of rabbit tibialis anterior muscle to chronic nerve stimulation. J Physiol 1983;338:1-9.

40. Ratnayake U, Quinn T, Daruwalla K, Dickinson H, Walker DW. Understanding the behavioural phenotype of the precocial spiny mouse. Behav Brain Res 2014;275:62-71. 